\title{
A Model for Phase Transition based on Statistical Disassembly of Nuclei at Intermediate Energies
}

\author{
G. Chaudhuri, S. Das Gupta, and M. Sutton \\ Physics Department, McGill University, Montréal, Canada H3A 2T8
}

(October 26, 2018)

\begin{abstract}
Consider a model of particles(nucleons) which has a two-body interaction which leads to bound composites with saturation properties. These properties are : all composites have the same density and the ground state energies of composites with $k$ nucleons are given by $-k W+\sigma k^{2 / 3}$ where $W$ and $\sigma$ are positive constants. $W$ represents a volume term and $\sigma$ a surface tension term. These values are taken from nuclear physics. We show that in the large $N$ limit where $N$ is the number of particles such an assembly in a large enclosure at finite temperature shows properties of liquid-gas phase transition. We do not use the two-body interaction but the gross properties of the composites only. We show that (a) the $p-\rho$ isotherms show a region where pressure does not change as $\rho$ changes just as in Maxwell construction of a Van der Waals gas, (b) in this region the chemical potential does not change and (c) the model obeys the celebrated Clausius-Clapeyron relations. A scaling law for the yields of composites emerges.

For a finite number of particles $N$ (upto some thousands) the problem can be easily solved on a computer. This allows us to study finite particle number effects which modify phase transition effects.

The model is calculationally simple. Monte-Carlo simulations are not
\end{abstract}


needed.

64.60-i,64.10+h,25.70Mn, 25.70Pq

Typeset using REVTEX 


\section{INTRODUCTION}

A very popular and highly successful model for collisions of two nuclei at intermediate energies (50 MeV to $100 \mathrm{MeV}$ per nucleon) is the following. Because of many collisions between nucleons, a statistical equilibrium is reached. The temperature rises. The system expands from normal density and composites are formed on the way to disassembly. As the system reaches between 3 to 6 times the normal volume, the interactions between the composites become unimportant (except for the long range Coulomb interaction) and one can do a statistical equilibrium calculation to obtain the yields of the composites at a volume which is called the freeze-out volume. Although the model is simple, actual realistic calculations based on the model are much harder. The nucleus is a finite system. It has two kinds of particles, neutrons and protons (generically termed nucleons). Protons carry charges and prevent large nuclei from being formed. For realistic treatment, the idea of a strict freeze-out volume has to be modified.

Here we consider the same physics but with the following simplifications: only one kind of particle is considered and the Coulomb interaction is neglected meaning arbitrarily large "nuclei" can be formed. The energy scale is MeV (million electron volt) and the length scale is $\mathrm{fm}\left(10^{-13} \mathrm{~cm}\right)$ so the salient features of nuclear physics are retained. The binding energy and the volume of a composite is proportional to the number of particles (nucleons) in the composite and have a surface tension proportional to the surface area. We show, with rather little effort, that the model leads to a first order phase transition as either the density the temperature or both are varied. The system has a region of liquid-gas coexistence where, as for the Maxwell construction of a Van der Waals gas, pressure remains constant when the density increases along the isotherm. In this region the chemical potential remains unchanged. As one traverses the path from the liquid phase to the gas phase the ClausiusClapeyron relationship is obeyed. For large systems, a scaling law for composites emerges: if we know the yields of composites for one large system, we know these for another large system. 
A more realistic version of this model has been used for Bevalac physics $(>250 \mathrm{MeV}$ per nucleon beam energy in the lab) by many authors more than twenty-five years ago. It is not possible to quote all the references but a review article [1] has a more complete list. The possibility of a phase transition was not considered as the collision energies were too high for the liquid phase and only very light composites could be produced.

Phase transitions in heavy ion collisions at intermediate energies became a topic of considerable interest starting from the mid-eighties and continues to be a central issue. There are many approaches and a large literature too numerous to list. We will refer here to only a few which closely follow the underlying physics considered here. The same model

as used here was adopted in [2] for finite nuclei. By extrapolation it was shown that the model leads to a first order phase transition. A brief application of this model is given in section VII. A grand canonical model was used in [3] which demonstrated a first order phase transition. The approach was quite different from what is used here. We use simpler, more traditional and numerical methods. Our results are similar but sufficiently different to warrant a full description. A discussion of Clausius-Clapeyron relations and a scaling law highlight some interesting physics.

The celebrated statistical Multifragmentation model (SMM) of Copenhagen [4], the microcanonical models of Gross and Randrup, Koonin $[5,6]$ use the same underlying physics as in this work. But the emphasis was on trying to be as close to to the actual nuclear situation as one can and thus the phase transition aspects are largely hidden.

\section{THE BASIC FORMULAE}

If we have $n_{a}$ particles of type $a, n_{b}$ particles of type $b, n_{c}$ particles of type $c$ etc. all enclosed in a volume $V$ and interactions between particles can be neglected, the grand partition function for this case can be written as

$$
Z_{g r}=\prod_{i=a, b, c \ldots} \exp \left(e^{\beta \mu_{i}} z_{i}\right)
$$


Here the $\mu_{i}$ is the chemical potential and $z_{i}$ the canonical partition function of one particle of type $i$. The average number of particles of type $i$ is given by $\partial\left(\ln Z_{g r}\right) / \partial\left(\beta \mu_{i}\right)$ :

$$
n_{i}=e^{\beta \mu_{i}} z_{i}
$$

It is possible that one of the species can be built from two other species. In reverse, a heavier species can also break up into two lighter species. If $\alpha$ number of particles of type $a$ can combine with $\beta$ number of particles of type $b$ to produce $\gamma$ number of particles of type $c$, then chemical equilibrium implies [7] that the chemical potentials of $a, b$ and $c$ are related by $\alpha \mu_{a}+\beta \mu_{b}=\gamma \mu_{c}$.

In our model we have $N$ nucleons in a volume $V$ but these nucleons can be singles or form bound dimers, trimers etc. Chemical equilibrium implies that a composite with $k$ bound nucleons has a chemical potential $k \mu$ where $\mu$ is the chemical potential of the monomer (nucleon). Thus our ensemble has monomers, dimers, trimers etc. upto some species with $k_{\max }$ bound nucleons where ideally $k_{\max } \rightarrow \infty$. For practical calculations, we use a finite value of $k_{\max }$. Most of the results shown here use $k_{\max }=2000$ although we have also done calculations with much larger values. Choosing $k_{\max }=2000$ does not mean that the total number of nucleons is 2000. The total number of nucleons can be infinite but the largest species allowed in the calculation (is somewhat artificially) limited to 2000. The total number of nucleons will be denoted by $N$ where $N$ is very large. The quantity $k_{\max }$ plays an essential role, setting $k_{\max }$ too low (for example 200 as shown in section VII) makes then liquid-gas transition disappear. An assembly with $\simeq 200$ particles or less does not display the typical behaviour of liquid-gas co-existence.

We now look into $z_{i}$, the partition function of one composite of $i$ nucleons. This factors into two parts, a traditional translation energy part and an intrinsic part: $z_{i}=z_{i}($ tran $) z_{i}($ int $)$ where

$$
\begin{aligned}
z_{i}(\operatorname{tran}) & =\frac{V}{h^{3}} \int \exp \left(-\beta p^{2} / 2 m_{i}\right) d^{3} p \\
& =\frac{V}{h^{3}}\left(2 \pi m_{i} T\right)^{3 / 2}
\end{aligned}
$$


The intrinsic part $z_{i}($ int $)$ of course contains the key to phase transition. If we regard each composite to exist only in a ground state with energy $e_{i}^{g r}$, then $z_{i}($ int $)=\exp \left(-\beta e_{i}^{g r}\right)$. We use $e_{i}^{g r}=-i W+\sigma i^{2 / 3}$ where nuclear physics sets $W=16 \mathrm{MeV}$ and $\sigma=18 \mathrm{Mev}$. This simple model itself will lead to the main results of this paper. Because of the surface term, energy per particle drops as $i$ grows. Let us denote by $F$ the free energy of the $N$ nucleons where $N$ is the total number of nucleons; $E$ be the energy and $S$, the entropy: $F=E-T S$. At finite temperature $F$ will go to its minimum value. The key issue is how the system of $N$ nucleons breaks up into clusters of different sizes as the temperature changes. At low temperature $E$ and hence $F$ minimises by forming very large clusters (liquid). But as the temperature increases $S$ will increase by forming larger number of clusters thus breaking up the big clusters. Gaseous phase will appear. How exactly this will happen requires calculation and we find that the system goes through a first order liquid-gas phase transition.

We used here a slightly more sophisticated model for $z_{i}($ int $)$. This does not make the calculation any harder (or alter the qualitative features) but makes it more realistic. We make the surface tension temperature dependent in conformity with usual parametrisation $[4] ; \sigma(T)=\sigma_{0}\left[\left(T_{c}^{2}-T^{2}\right) /\left(T_{c}^{2}+T^{2}\right)\right]^{5 / 2}$

Here $\sigma_{0}=18 \mathrm{MeV}$ and $T_{c}=18 \mathrm{MeV}$. At $T=T_{c}$ surface tension vanishes and we have a fluid only. For us this is unimportant as our focus will be the temperature range 3 to 8 $\mathrm{MeV}$.

In computing the partition function $z_{i}($ int $)$ we include not just the ground state but also excited states of the composite in an approximate fashion. We should compute $z_{i}($ int $)=$ $\exp \left(-\beta e_{i}^{g r}\right)+\int \sum g_{i}(e) \exp (-\beta e)$. Here $e>e_{i}^{g r}$ and $g_{i}(e)$ is the density of excited states of this particular composite. Instead of trying to calculate $z_{i}($ int $)$ by performing the sum and integral we use a well-known trick. Utilise the relation $z_{i}(i n t)=\exp \left(-f_{i}(\right.$ int $\left.) / T\right)$ where $f_{i}($ int $)=e_{i}^{T}-T s_{i}$ and now use the Fermi-gas formula for the nucleus with $i$ nucleons (approximately correct and widely used at intermediate temperature). This gives $e_{i}^{T}=$ $e_{i}^{g r}+i T^{2} / \epsilon_{0}$. This is similar to electron gas at finite temperature (excitation energy goes like $T^{2}$ ) except that in nuclear physics the value of $\epsilon_{0}$ is $\simeq 16 \mathrm{MeV}$. The intrinsic entropy of 
the nucleus at this temperature is $2 i T / \epsilon_{0}$. The expression for $z_{i}(i n t)$ is now complete and easily tractable.

Let us now summarise the relevant equations. For $k=1$ (the nucleon which has no excited states)

$$
n_{1}=\frac{V}{h^{3}}(2 \pi m T)^{3 / 2} \exp (\mu / T)
$$

and for $k>1$

$$
n_{k}=\frac{V}{h^{3}}(2 \pi m T)^{3 / 2} k^{3 / 2} \exp \left[\left(\mu k+W k+k T^{2} / \epsilon_{0}-\sigma(T) k^{2 / 3}\right) / T\right]
$$

Here $n_{k}$ is the average number of composites with $k$ nucleons. In the rest of the paper, for brevity, we will omit the qualifier "average". It is always implied.

A useful quantity is the multiplicity defined as

$$
M=\sum_{k=1}^{k_{\max }} n_{k}
$$

The number of nucleons bound in a composite with $k$ nucleons is $k n_{k}$ and obviously $N=$ $\sum_{k=1}^{k_{\max }} k n_{k}$. The pressure is given by

$$
p=\sum_{k=1}^{k_{\max }} \frac{n_{k}}{V} T
$$

Quantities like $N, V, n_{k}$ are all extensive variables. These equations can all be cast in terms of intensive variables like $N / V=\rho, n_{k} / N$ etc so that we can assume both $N$ and $V$ approach very large values and fluctuations in the number of particles can be ignored. Thus for a given temperature and density we solve for $\mu$ using

$$
\rho=\frac{(2 \pi m T)^{3 / 2}}{h^{3}}\left(\exp (\mu / T)+\sum_{k=2}^{k_{\max }} k^{5 / 2} \exp \left[\left(\mu k+W k+k T^{2} / \epsilon_{0}-\sigma(T) k^{2 / 3}\right) / T\right]\right)
$$

The sum rule $N=\sum_{k=1}^{k_{\max }} k n_{k}$ changes to $1=\sum k n_{k} / N$.

From what we have described so far it would appear that $V$ in eqs.(3), (4) and (6) is the freeze-out volume $V$, the volume to which the system has expanded. Actually if the freeze-out volume is $V$ then in these equations we use $\tilde{V}$ which is close to $V$ but less. The 
reason for this is the following. To a good approximation a composite of $k$ nucleons is an incompressible sphere with volume $k / \rho_{0}$ where the value of $\rho_{0}$ is $\simeq 0.16 \mathrm{fm}^{-3}$. The volume available for translational motion (eq.(3)) is then $\tilde{V}=V-V_{\text {excluded }}$ where we approximate $V_{\text {excluded }} \simeq N / \rho_{0}=V_{0}$ the normal volume of a nucleus with $N$ nucleons. Similar corrections are implicit in Van der Waals equation of state. This is meant to take care of hard sphere interactions between different particles. This answer is approximate. The correct answer is multiplicity dependent. The approximation of non-interacting composites in a volume gets to be worse as the volume decreases. We restrict our calculation to volumes $V$ greater than $2 V_{0}$. This is how the calculations reported in the next section proceed. We choose a value of $V_{0} / V=\rho / \rho_{0}$ from which $V_{0} / \tilde{V}=\tilde{\rho} / \rho_{0}=\rho /\left(\rho_{0}-\rho\right)$ is deduced. This value of $\tilde{\rho}$ is used in eq.(8) to calculate $\mu$ and all other quantities. We plot results as function of $\rho / \rho_{0}$. If we plotted them as function of $\tilde{\rho} / \rho_{0}$ the plot would shift to the right.

Calculations in [3] are continued beyond the limit $\rho / \rho_{0}=0.5$. They find one can identify a critical point at $T=T_{c}=18.0 \mathrm{MeV}, \rho / \rho_{0}=1$ and $p_{c}=\infty$. At very high pressure the model should break down: $z_{k}$ (int) must change at such high pressure although nuclear physics says that nuclei being highly incompressible moderate pressures should leave the internal partition functions relatively unchanged. Another way of saying this is that interaction between composites should be taken into account for $V \simeq V_{0}$.

\section{THE $P-\rho$ CURVES FOR ISOTHERMALS}

For a given temperature $T$ and $\rho$ we solve for $\mu$ and then pressure. This is plotted in Fig. 1. For each isotherm shown the pressure rises rapidly at first with $\rho$ but then flattens out. The flattening depends upon the value of $k_{\max }$. For low $k_{\max }$ (shown in section VII) there will not be any flattening. The value of $k_{\max }$ used in Fig. 1 is 2000 . There is still a very slight rise in $p$ (not discernible in the Figure). The figure empirically allows us to designate two regions: a purely gas phase where the pressure rises with density and a liquidgas co-existence phase where the volume changes but the pressure is nearly stationary. One 
way of seeing this is that $p=T(M / \tilde{V})$. As $\tilde{V}$ decreases so does $M$ so as to compensate in the co-existence region. Fig. 1 also shows that in the gas phase, the chemical potential rises rapidly with density but then flattens out in the co-existence phase.

A discussion about $\mu$ dependence in the co-existence region is in order here. For $k_{\max }$ large $\rho$ is much more sensitive to $\mu$ than pressure ( $\rho$ has weighting of $k^{5 / 2}$, eq.(8) whereas $p$ has $k^{3 / 2}$ weighting (eqs. (5) and (7)). For very large $k_{\max }$ an infinitesimal change in $\mu$ will lead to a finite change in $\rho$ but only a very small change in $p$. In the limit $k_{\max } \rightarrow \infty$ we will reach ideal liquid gas phase transition: no change of $\mu$ in the coexistence region and no change of pressure. This is demonstrated in section VI.

\section{WHAT CONSTITUTES THE GAS AND WHAT CONSTITUTES THE LIQUID?}

As an example, at fixed temperature $7 \mathrm{MeV}$ we show in Fig. 2 the distribution of composites (a) in the pure gas region $\left.\left(\rho / \rho_{0}=0.12\right)\right)$ and $(\mathrm{b})$ in the co-existence region $\left(\rho / \rho_{0}=0.22\right)$. In the gas phase the sum rule $\sum k n_{k} / N=1$ is exhausted well before we reach 50 . There are no heavy composites. In the co-existence region there are light particles $(k \leq 40)$, then nothing for a long range of $k$, and then there are heavy particles with $k$ between 1800 to $k_{\max }=2000$ (the figure shows the population of $k=1900$ to $k_{\max }=2000$ ). A safe functional definition for the gas phase is all composites between $k=1$ to $k=100$ and for the liquid phase all composites between $k_{\max }-300$ to $k_{\max }$. Thus both the liquid and the gas phases are quite complicated; consisting of not one or two but many species although they are all made up of the same elemental nucleon.

\section{THE CLAUSIUS-CLAPEYRON RELATIONS}

In the co-existence region the pressure is a rapidly increasing function of the temperature. In Fig. 1 these are the flat regions shown for three temperatures (dash-dot $6 \mathrm{MeV}$, solid 7 
$\mathrm{MeV}$ and dash 7.5 MeV). The Clausius-Clapeyron relation for liquid-gas phase transition provides an equation for the rate of change [7]:

$$
\frac{d p}{d T}=\frac{\Delta s}{\Delta v}
$$

where $\Delta s$ can be taken to be the change of entropy per unit mass and $\Delta v$ the corresponding change in volume as matter moves across phase transition. We can take these changes to be per nucleon. The following substitutions are made: $\Delta s=L / T$ ( $L$ is the latent heat) and $\Delta v=v_{\text {gas }}-v_{\text {liq }}$. The standard approximation now is $v_{\text {gas }}>>v_{\text {liq }}, v_{\text {gas }}=1 / \rho_{\text {gas }} \simeq T / p$ and thus

$$
\frac{d p}{d T} \simeq \frac{L p}{T^{2}}
$$

If we make the assumption that $L$ is nearly independent of temperature then the equation integrates out to give

$$
\ln p=\ln p_{0}-L / T
$$

This does not work well in our case (Fig. 3): $\ln p$ is not a linear function of $\beta=1 / T$. We can discard the assumption that $L$ is constant and instead use eq.(10) to get an idea of $L$ using values of $d p / d T, p$ and $T$ from Fig. 1 . If this is done then at $6 \mathrm{MeV}$ temperature, the value of $L$ turns out to be $54 \mathrm{MeV}$ and at $7 \mathrm{MeV}$ temperature this reaches $70 \mathrm{MeV}$. Since the binding energy per particle for an infinite cluster is $16 \mathrm{MeV}$, these values are clearly unacceptably high.

Let us ask what went wrong in going from eq.(9) to eq.(11). The passage from eq.(10) to eq.(11) assumed that the latent heat is independent of temperature. We will show that this is approximately correct. However, the approximation $v_{\text {gas }} \simeq T / p$ is very inaccurate and depending upon the temperature, corrections due to $v_{l i q}$ can be significant. When all this is taken into account eq.(9) is satisfied remarkably well. We elaborate first on the latent heat. For definiteness fix on the isothermal at $7 \mathrm{MeV}$. From Fig. 1 we can determine the density at which the system enters the purely gaseous phase and its energy per particle from $\sum n_{k} e_{k} / N$ 
where $e_{k}=(3 / 2) T+e_{k}(i n t)$. Here $e_{k}(i n t)$ consists of volume energy (which is negative), surface energy and as well contributions from excited states. The expressions are given in section II. The nucleons are passing from a liquid state (from a composite with $k \simeq 2000$ ) to the gaseous phase. The energy per particle in the liquid phase was taken from a composite of 1950 particles. Reasonable variation around this number will only change the calculated value slightly. Latent heat per particle calculated is $12.66 \mathrm{MeV}$ at temperature $6 \mathrm{MeV}$ and $11.55 \mathrm{MeV}$ at $7.5 \mathrm{MeV}$.

By far the major error is in assuming that $v_{\text {gas }} \simeq T / p$. The pressure is given by $p=$ $(M / \tilde{V}) T$ and not $(N / \tilde{V}) T$ where $M$ is the multiplicity and $N$ of course the total number of particles. Thus $p=(M / N) T / v_{\text {gas }}$. The factor $(M / N)$ when the system just turns into a pure gas phase is 0.276 at $\mathrm{T}=6.0 \mathrm{Mev}, 0.194$ at $7.0 \mathrm{MeV}$ and 0.152 at $7.5 \mathrm{MeV}$. Writing $\alpha$ for $M / N$ we find that eq.(10) should be rewritten as

$$
\frac{d p}{d T}=\frac{L p}{T^{2} \alpha\left[1-p /\left(\rho_{0} \alpha T\right)\right]}
$$

where we have used the fact that $v_{l i q}=1 / \rho_{0}$. As an example, at $7 \mathrm{MeV} \frac{d p}{d T}=0.0535 \mathrm{fm}^{-3}$ from Fig. 1 and $0.0530 \mathrm{fm}^{-3}$ estimated from formula (12). At $6 \mathrm{MeV}$ the corresponding numbers are $0.0129 \mathrm{fm}^{-3}$ and $0.0108 \mathrm{fm}^{-3}$ respectively.

\section{THE LIMIT LARGE $K_{M A X}$.}

We will now consider changes in the values of various quantities as we change from one large value of $k_{\max }$ to another large value of $k_{\max }$. For definiteness we will concentrate on one isothermal, for example the $T=7 \mathrm{MeV}$ case. In Fig. 1 we have two regions: pure gas phase and the co-existence phase. The pure gas phase is trivial. Nothing changes as we go from one large $k_{\max }$ to another; $k_{\max }=2000$ is large enough in this case. It is easy to see why results become insensitive to changes in the value of $k_{\max }$. In the gas phase there is no population in high $k$ composites so it does not matter whether the summation stops at a given high value of $k_{\max }$ or another high value of $k_{\max }$. The situation is more complicated 
but also more interesting in the co-existence region as we have population both at the lower end and the higher end of $k$.

In Fig. 4 we plot the values of $\mu$ and pressure calculated for $k_{\max }$ in the range $k_{\max }=2000$ to 5000. This is done at a fixed value of $\rho / \rho_{0}=0.3$ which is in the co-existence region. As shown in the figure, both curves are well fit by a parametrisation $a+b \exp \left(-c k_{\max }\right)$ where $k_{\max } \geq 2000$, with values given in the caption. This means that within the accuracy with which this calculation was carried out, the values of $\mu$ and pressure in the infinite $k_{\max }$ limit are $-18.504 \mathrm{MeV}$ and $0.0294 \mathrm{MeV} \mathrm{fm}^{-3}$ respectively.

A similar calculation as above was done for $\rho / \rho_{0}=0.4$. The fitted values of $a, b$ and $c$ for $\mu$ were $-18.504 \mathrm{MeV}, 0.33892 \mathrm{MeV}$ and 0.0003197 respectively. For pressure, the parameters were $0.0294 \mathrm{MeV} \mathrm{fm}^{-3}, 0.007633 \mathrm{MeV} \mathrm{fm}^{-3}$ and 0.0003906 respectively. Note that the extrapolation demonstrates that that neither $\mu$ nor the pressure change in the co-existence region in the limit $k_{\max } \rightarrow \infty$. This firmly establishes the present model as a model of liquid-gas phase transition as was stated in section III.

Lastly we want to estabish a scaling law. Given the fractional occupation $f_{k}\left(k_{\max }\right)=$ $k n_{k} / N$ for a large value of $k_{\max }$, do we know the fractional occupation $f_{k^{\prime}}\left(k_{\max }^{\prime}\right)$ for another large value of $k_{\max }$ ? Based on the discussion, so far we expect that if one is in the purely gas phase $f_{k}\left(k_{\max }\right)=f_{k}\left(k_{\max }^{\prime}\right)$ and this is indeed the case.

In the co-existence phase a lowest order approximation is based on the following approximation. We expect the fractional occupation to match near the beginning ( $k$ small), near the end (near $k \leq k_{\max }$ and $k^{\prime} \leq k_{\max }^{\prime}$ ) and in between there is almost no occupation. Thus for $k$ small $f_{k}\left(k_{\max }\right) \simeq f_{k}\left(k_{\max }^{\prime}\right)$ and near the high end $f_{k}\left(k_{\max }\right) \simeq f_{k^{\prime}}\left(k_{\max }^{\prime}\right)$ where $k_{\max }-k=k_{\max }^{\prime}-k^{\prime}$. This is not very accurate but an accurate representation for low $k$ is given using the parametrisation:

$$
\ln f_{k}\left(k_{\max }\right)=\ln f_{k}\left(k_{\max }^{\prime}\right)+\frac{k}{T} b\left[\exp \left(-c k_{\max }\right)-\exp \left(-c k_{\max }^{\prime}\right)\right]
$$

An equation relating the large clusters can also be written down, but the functional form is quite complicated. 


\section{SMALL SYSTEMS: AN EXACT CANONICAL MODEL SOLUTION}

The model can be solved when the number of particles $N$ is finite. Extensive use of the canonical model has been made to fit experimental data [8] so just an outline will be presented for completeness. Among other applications, the canonical model can be used to study finite particle number effects on phase transition characteristics.

Consider again $N$ identical particles in an enclosure $V$ and temperature $T$. These $N$ nucleons will combine into monomers, dimers, trimers etc. The partition function of the system in the canonical ensemble can be written as

$$
Q_{N}=\sum \prod_{i} \frac{\left(z_{i}\right)^{n_{i}}}{n_{i} !}
$$

Here $z_{i}$ is the one particle partition function of a composite which has $i$ nucleons. We already encountered $z_{i}$ in section II: $z_{i}=z_{i}(\operatorname{tran}) z_{i}($ int $)$ with $z_{i}(\operatorname{tran})$ and $z_{i}($ int $)$ given in detail. Other forms for $z_{i}$ can be used in the method outlined here. The summation in eq.(14) is over all partitions which satisfy $N=\sum i n_{i}$. The summation is non-trivial as the number of partitions which satisfy the sum is enormous. We can define a given allowed partition to be a channel. The probablity of the occurrence of a given channel $P(\vec{n}) \equiv P\left(n_{1}, n_{2}, n_{3} \ldots\right)$ is

$$
P(\vec{n})=\frac{1}{Q_{N}} \prod \frac{\left(z_{i}\right)^{n_{i}}}{n_{i} !} .
$$

The average number of composites of $i$ nucleons is easily seen from the above equation to be

$$
\left\langle n_{i}\right\rangle=z_{i} \frac{Q_{N-i}}{Q_{N}}
$$

Since $\sum i n_{i}=N$, one readily arrives at a recursion relation [9]

$$
Q_{N}=\frac{1}{N} \sum_{k=1}^{N} k z_{k} Q_{N-k}
$$

For one kind of particle, $Q_{N}$ above is easily evaluated on a computer for $N$ as large as 3000 in matter of seconds. It is this recursion relation that makes the computation so easy in the model. Of course, once one has the partition function all relevant thermodynamic 
quantities can be computed. For example, eq. (7) still gives the expression for pressure although one could correct for the center of mass motion by reducing the multiplicity by 1 : $p=T(M-1) / \tilde{V}$. The chemical potential can be calculated from $\mu=F(N)-F(N-1)$ where the free energy is $F(N)=-T \ln Q_{N}$ which is readily available from the calculation.

In Fig. 5 we show an example of the canonical model calculation. The temperature is $6 \mathrm{MeV}$. The number of particles $N$ is 200 . The value of the largest allowed cluster $k_{\max }$ is also 200. This would be a typical nuclear physics case. In the same figure we also show the result of a grand canonical calculation with the same $k_{\text {max }}$ (of course for the grand canonical $N$ is very large). At small density the results are the same but they become different at larger densities. In the canonical result there is a small region where $d p / d \rho$ is negative. This is a finite particle number effect since for large $N$ (grand canonical result) any negative

compressibilty disappears. Negative compressibility can lead to negative value for $c_{p}$ [8]. The grand canonical result shows that for $k_{\max }=200$ typical liquid-gas co-existence is not found and there is no region where $p$ is constant when the density changes.

\section{DISCUSSION}

Results in section III to section VI show that the model of excited matter breaking up into clusters with saturation properties leads to a first order phase transition. This has relevance to heavy ion collisions at intermediate energy but may have significance in other areas of physics as well. This model for first order phase transitions is extremely easy to implement. A very significant advantage of the model is that it can be solved not only in the thermodynamic limit (large $N$ ) but also for a finite number of particles. Thus one can study how observables change as one progresses from small to large systems.

We like to end this discussion by noting that in spite of a very different approach that is adopted here to arrive at the key equations 5, 7 and 8, formally the pressure and density equations have the same structure as those encountered in the well-known Mayer cluster expansion [10]. These are $p=\frac{(2 \pi m T)^{3 / 2}}{h^{3}} \sum_{k=1}^{\infty} \exp (\beta \mu k) b_{k}$ and $\rho=\frac{(2 \pi m T)^{3 / 2}}{h^{3}} \sum_{k=1}^{\infty} \exp (\beta \mu k) k b_{k}$. 
Here instead of the cluster integral $b_{k}$ we have $k^{3 / 2} z_{k}($ int $)$.

\section{ACKNOWLEDGEMENT}

One of the authors (S.D.G) is indebted to Aram Mekjian for collaboration over many years in the subject of heavy ion collisions. Many of the ideas amplified in this work owe their origin to these collaborations. The same author is also indebted to Champak Baran Das for past joint ventures. This work is supported in part by the Natural Sciences and Engineering Research Council of Canada and in part by the Quebec Department of Education. 


\section{REFERENCES}

[1] S. Das Gupta, and A. Z. Mekjian, Phys. Rep.72, 131 (1981)

[2] S. Das Gupta and A. Z. Mekjian, Phys. Rev. C57, 1361 (1998)

[3] K. A. Bugaev, M. I. Gorenstein, I. N. Mishustin, and W. Greiner, Phys. Rev C62, 044320 (2000)

[4] J. P. Bondorf, A. S. Botvina, A. S. Iljinov, I. N. Mishustin and K. Sneppen, Phys. Rep. $257,133(1995)$

[5] D. H. Gross, Phys. Rep. 279, 119 (1997) 1040 (1995)

[6] J. Randrup, and S. E. Koonin, Nucl. Phys. A471, 355c (1987)

[7] F. Reif, Fundamentals of statistical and thermal physics(McGraw Hill, New York, 1965)Chapter 8.

[8] C. B. Das, S. Das Gupta, W. G. Lynch, A. Z. Mekjian, and M. B. Tsang, Phys. Rep. 406, 1, (2005)

[9] K. C. Chase, and A. Z. Mekjian, Phys. Rev C52,R2339, (1995)

[10] R. K. Pathria, Statistical Mechanics(Pergamon press, Toronto, 1980)Chapter 9. 


\section{FIGURES}
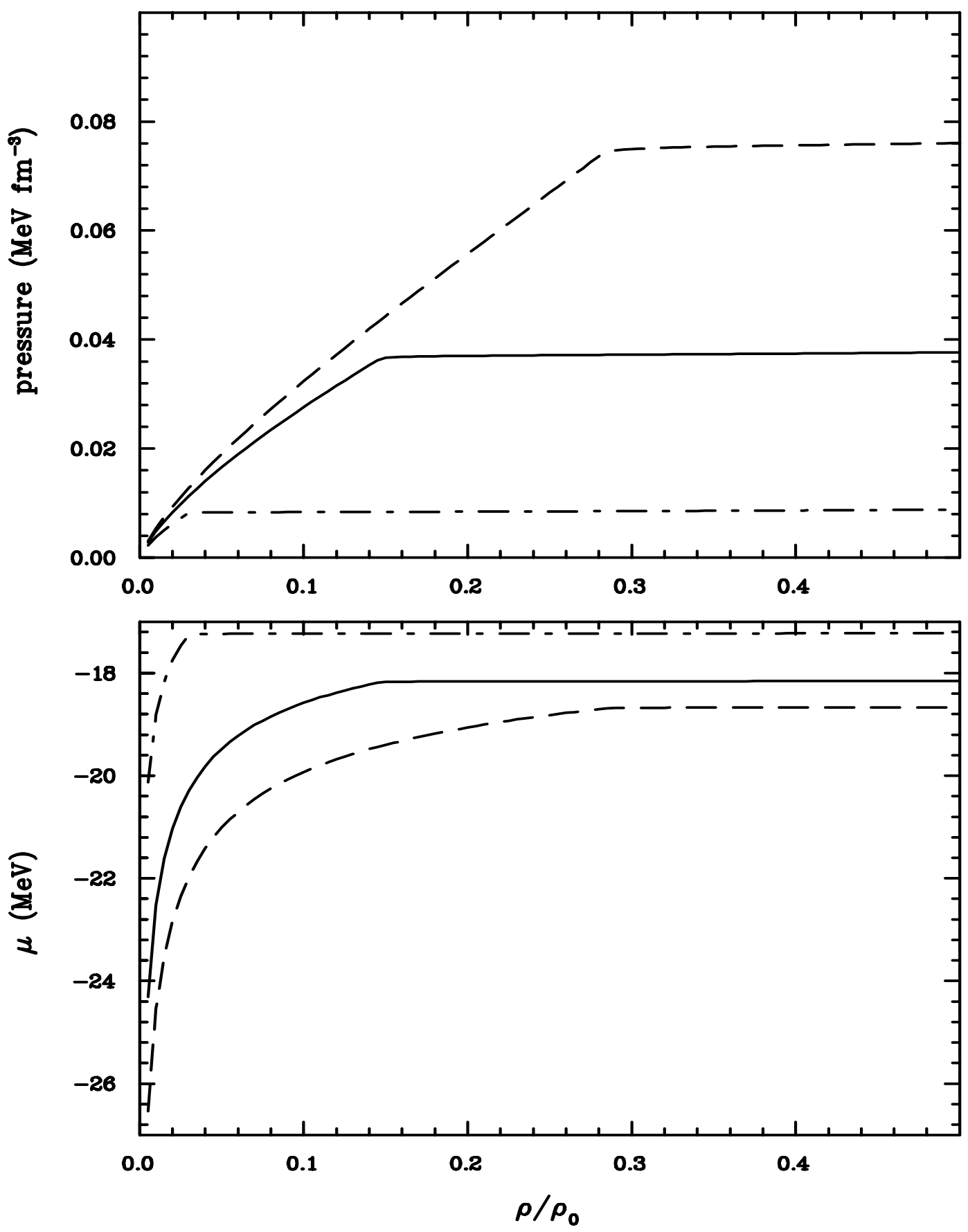

FIG. 1. Behavior of pressure $p$ and chemical potential $\mu$ against $\rho / \rho_{0}$ for 3 different temperatures : dash $(7.5 \mathrm{MeV})$, solid $(7 \mathrm{MeV})$ and dash-dot $(6 \mathrm{MeV})$. We identify as purely gas phase the region where the pressure and the chemical potential $\mu$ rise with density and the co-existence region where they remain constsnt. 

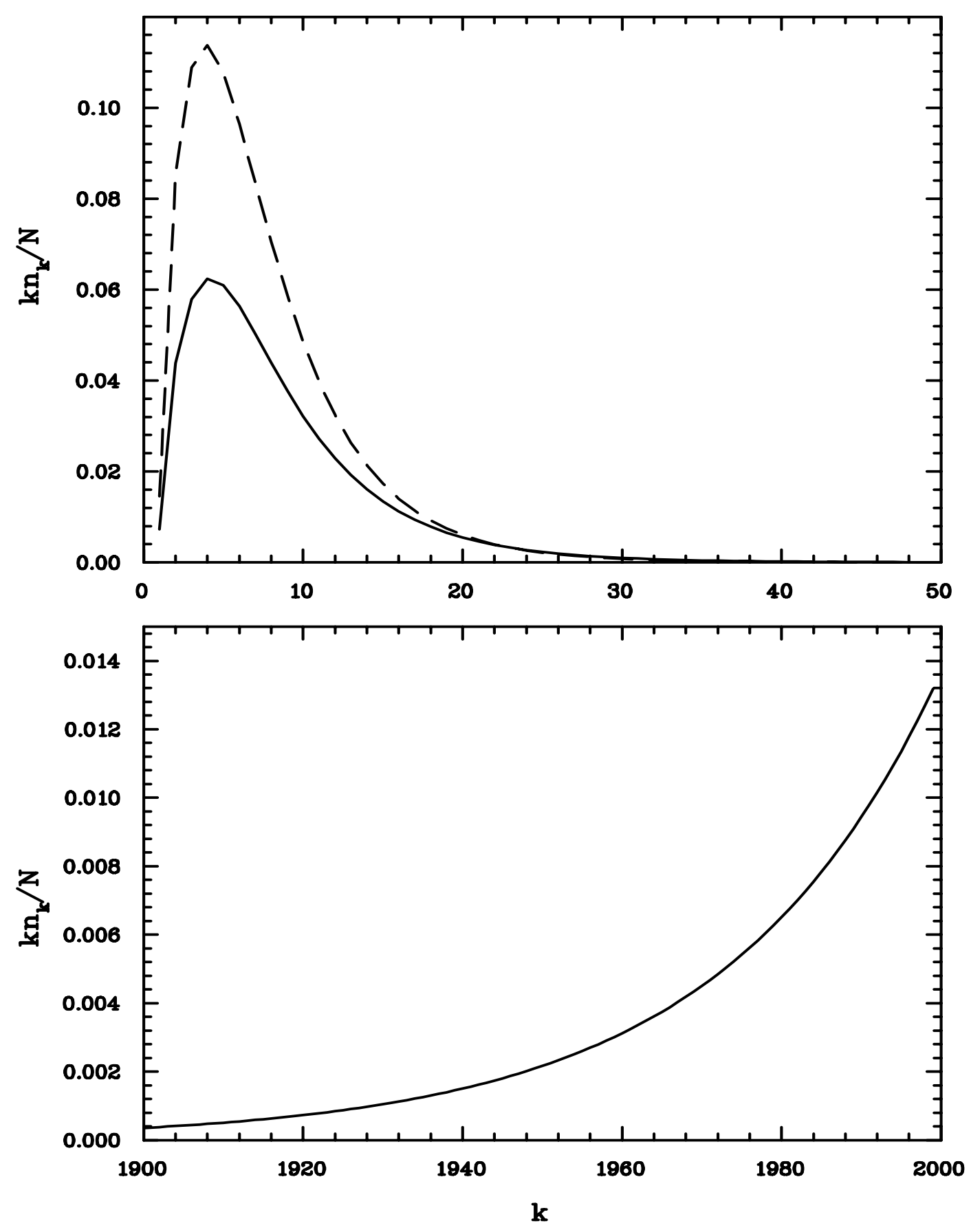

FIG. 2. At temparature $7 \mathrm{MeV}$ yields of composites at two densities $\rho / \rho_{0}=0.12$ (gas phase) and $\rho / \rho_{0}=0.22$ (the co-existence phase). For the first case there are no heavy composites (dashed line). The sum rule $\sum k n_{k} / N=1$ is already satisfied to good accuracy by $k=40$. In the second case (solid line) there are light particles (less than 50 nucleons) and there are heavy particles (greater than 1800 particles). Together these exhaust the sum rule. In $k$ space there is a huge gap for particles between large and small. The occupation number in this region is very close to 0 . 


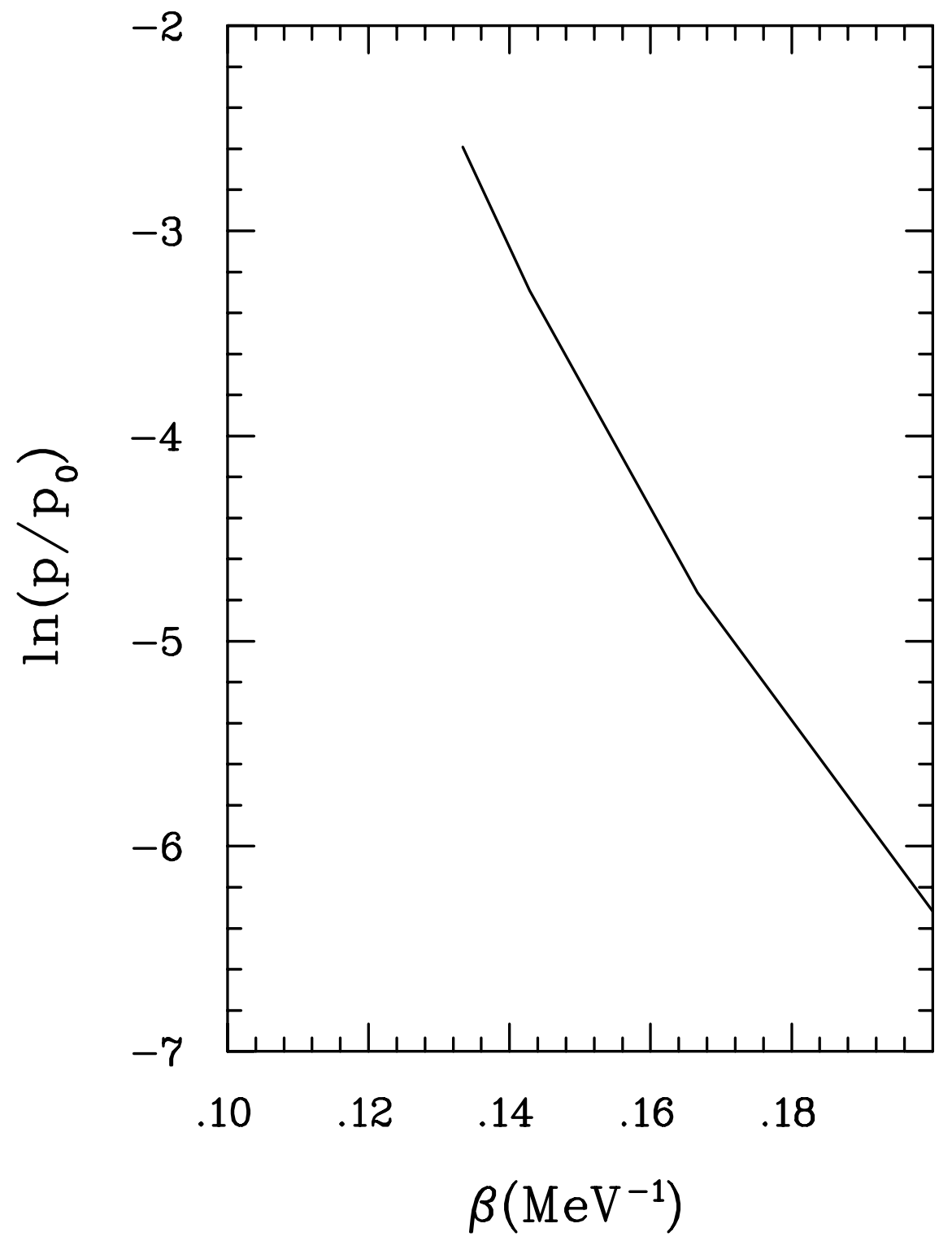

FIG. 3. A plot of $\ln (p)$ against the inverse of temperature. The relationship is not linear. Here $p_{0}$ is $1 \mathrm{MeV} \mathrm{fm}^{-3}$. 

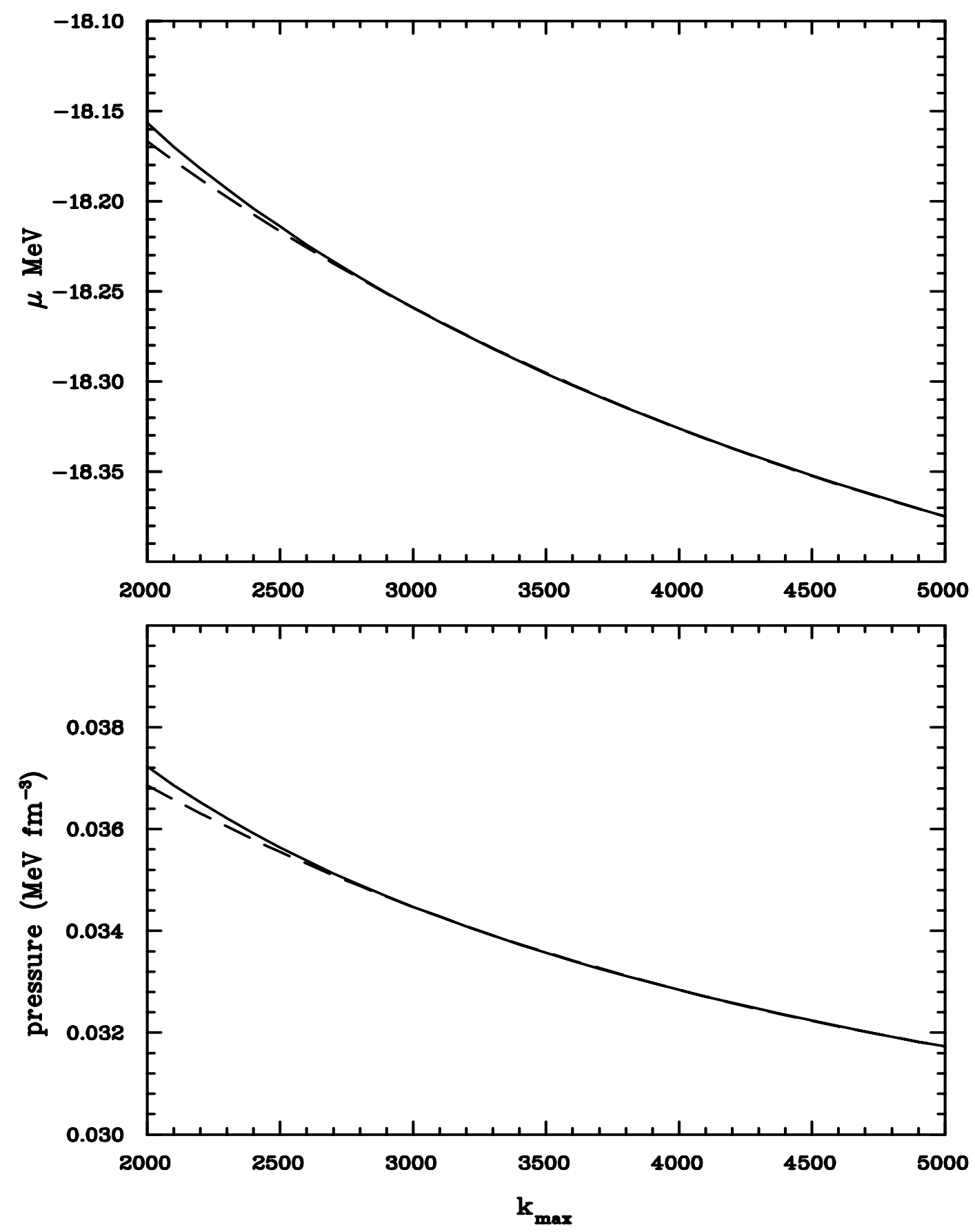

FIG. 4. The solid curve in the upper panel is a plot of $\mu$ against $k_{\max }$ in the range of $k_{\max }=2000$ to 5000 with density at $\rho / \rho_{0}=0.3$ and temperature $7 \mathrm{MeV}$. The dashed curve is a fit with the parametrisation $a+b \exp \left(-c k_{\max }\right)$. The values of the fit parameters are $a=-18.504 \mathrm{MeV}$, $b=0.33748 \mathrm{MeV}$ and $c=0.0003842$. Similar quantities for pressure in the lower panel. The fit parameters are $a=0.0294 \mathrm{MeV} \mathrm{fm}^{-3}, b=0.007503 \mathrm{MeV} \mathrm{fm}^{-3}$ and $c=0.0003842$. Similar curves for $\rho / \rho_{0}=0.4$ yield equally good fit and give the same values for $a$ but different values for $b$ and $c$. 

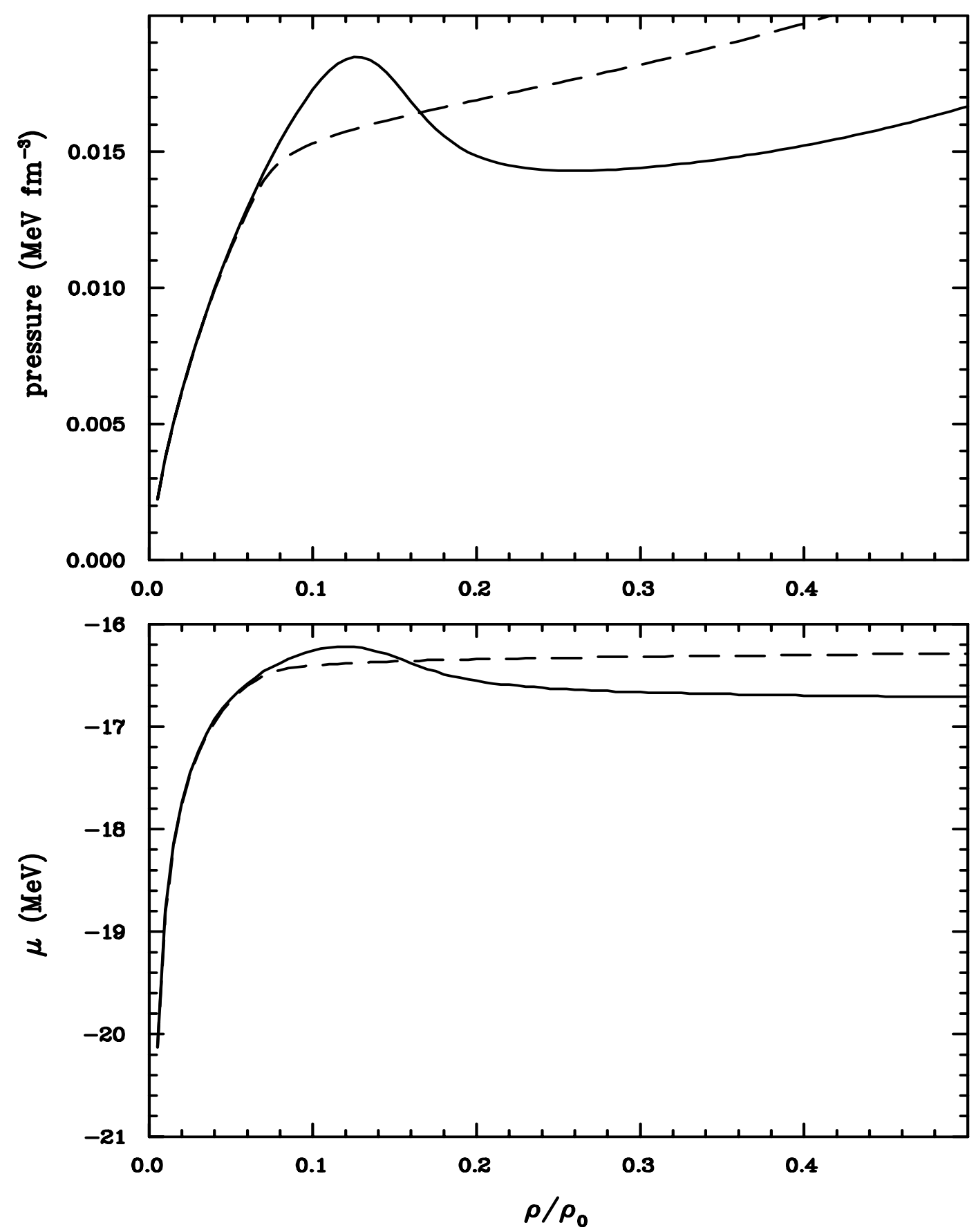

FIG. 5. The solid curve in the upper panel is a plot of pressure against density in the canonical model. The number of particles is $N=200$ exactly and $k_{\max }$ is also 200 . Note that there is a region of negative compressbilty. The dashed curve is the grand canonical result with the same $k_{\max }$. The two curves coincide at low density. Note that in the grand canonical model the increase of pressure with density goes down later but never disappears for this low $k_{\max }$. The lower panel compares the chemical potentials. 